\title{
Differential diagnosis of usual interstitial pneumonia: when is it truly idiopathic?
}

\author{
Wim A. Wuyts ${ }^{1}$, Alberto Cavazza², Giulio Rossi ${ }^{3}$, Francesco Bonella ${ }^{4}$, \\ Nicola Sverzellati ${ }^{5}$ and Paolo Spagnolo ${ }^{6}$
}

\begin{abstract}
Affiliations: 'Unit for Interstitial Lung Diseases, Dept of Pulmonary Medicine, University Hospitals Leuven, Leuven, Belgium. ${ }^{2}$ Operative Unit of Pathology, S. Maria Nuova Hospital, Reggio Emilia, Italy. ${ }^{3}$ Section of Pathology, University Hospital of Modena, Modena, Italy. "Section of Diagnostic Imaging, Dept of Surgery, University of Parma, Parma, Italy. ${ }^{5}$ Interstitial and Rare Lung Disease Unit, Ruhrlandklinik, University Hospital, University of Duisburg-Essen, Germany. ${ }^{6}$ Medical University Clinic, Canton Hospital Baselland and University of Basel, Liestal, Switzerland.
\end{abstract}

Correspondence: Paolo Spagnolo, Medical University Clinic, Canton Hospital Baselland, and University of Basel, Rheinstrasse 26, 4410 Liestal, Switzerland. E-mail: paolo.spagnololaksbl.ch

ABSTRACT Idiopathic pulmonary fibrosis (IPF), the most common and lethal of the idiopathic interstitial pneumonias, is defined by a radiological and/or pathological pattern of usual interstitial pneumonia (UIP). However, UIP is not synonymous with IPF as other clinical conditions may be associated with UIP, including chronic hypersensitivity pneumonitis, collagen vascular disease, drug toxicity, asbestosis, familial IPF and Hermansky-Pudlak syndrome. Differentiating IPF (“idiopathic UIP”) from conditions that mimic IPF ("secondary UIP") has substantial therapeutic and prognostic implications.

A number of radiological and histological clues may help distinguish IPF from other conditions with a UIP pattern of fibrosis, but their appreciation requires extensive expertise in interstitial lung disease as well as an integrated multidisciplinary approach involving pulmonologists, radiologists and pathologists. In addition, multidisciplinary discussions may decrease the time to initial IPF diagnosis and, thus, enable more timely management. This concept was strongly emphasised by the 2011 ATS/ERS/JRS/ALAT guidelines.

This article highlights, with the aid of a clinical case, the difficulties in making a diagnosis of IPF in clinical practice. Yet, an accurate diagnosis is critical, particularly given the availability of drugs that may reduce the pace of functional decline and disease progression in IPF.

0 @ERSpublications

Differentiating between idiopathic (IPF) and secondary UIP has crucial therapeutic and prognostic implications http://ow.ly/yqy27

\section{Clinical case}

In June 2012, a 62-year-old man presented to his primary care physician with slowly progressive shortness of breath and dry cough lasting for almost 1 year. He also reported fatigue, heartburn and mild weight loss. He was initially treated with antibiotics, inhaled bronchodilators and a short course of steroids without significant improvement in symptoms. His chest radiograph showed reticular infiltrates with bibasilar predominance consistent with an interstitial process. At this stage, the patient was referred to our clinic for further evaluation. He was, at that time, a current smoker of 60 pack-years and had a history of atrial

Received: June 102014 | Accepted: June 152014

Support statement: Wim A. Wuyts is a Senior Clinical Investigator of the Research Foundation - Flanders (grant 1.8.325.12N).

Conflict of interest: Disclosures can be found alongside the online version of this article at err.ersjournals.com

Provenance: Publication of this peer-reviewed article was sponsored by InterMune International AG, Muttenz, Switzerland (article sponsor, European Respiratory Review issue 133).

Copyright OERS 2014. ERR articles are open access and distributed under the terms of the Creative Commons Attribution Non-Commercial Licence 4.0. 
fibrillation (diagnosed in 2004) for which he took $200 \mathrm{mg} \cdot \mathrm{day}^{-1}$ amiodarone. He denied significant occupational exposures (he worked as sales representative), allergies, recent trips abroad or a family history of chronic respiratory diseases, although his mother had rheumatoid arthritis. He was exposed to parrots for a period of almost 5 years, until 1995. Chest auscultation revealed bibasilar end-inspiratory fine crackles (velcro-like). Clinical examination was otherwise unremarkable. The patient denied joint pain, stiffness or swelling, Raynaud's phenomenon, or other signs or symptoms suggestive of an underlying connective tissue disease (CTD). Lung function tests revealed a restrictive defect (forced vital capacity $68 \%$ predicted) with a moderately reduced diffusing capacity of the lung for carbon monoxide (DLCO; $42 \%$ pred). Blood test, CTD serology (including rheumatoid factor, anti-cyclic citrullinated peptide, and anti-nuclear antibody titre and pattern) and hypersensitivity pneumonitis (HP) precipitins panel were negative. High-resolution computed tomography (HRCT) of the chest showed bilateral subpleural reticular abnormalities without traction bronchiectasis or honeycombing but also without features "inconsistent" with the usual interstitial pneumonia (UIP) pattern. This combination of findings defines a possible UIP pattern (fig. 1), which, according to the current American Thoracic Society/ European Respiratory Society/Japanese Respiratory Society/Latin American Thoracic Association guidelines, requires further diagnostic evaluation [1]. Bronchoalveolar lavage (BAL) showed an increased total cell count $\left(240\right.$ cells $\left.\cdot \mu \mathrm{L}^{-1}\right)$ as well as an increased lymphocyte $(18 \%)$ and neutrophil (7\%) count; transbronchial biopsy displayed nonspecific findings. The patient, therefore, underwent a surgical lung biopsy (SLB), which was consistent with a UIP pattern (fig. 2). Based on clinical, radiological and pathological data, a diagnosis of idiopathic pulmonary fibrosis (IPF) was made. The patient started pirfenidone therapy and was referred for lung transplant evaluation.

\section{Challenges in diagnosing IPF in clinical practice}

This case highlights some of the difficulties that are encountered in clinical practice when facing patients with suspected IPF.

IPF is a chronic, progressive fibrotic interstitial pneumonia of unknown origin, limited to the lung and occurring primarily in older adults. The disease should be suspected particularly in male current or exsmokers $>60$ years of age with unexplained chronic exertional dyspnoea. By contrast, IPF is sufficiently uncommon in individuals $<50$ years of age to mandate a vigorous search for known (e.g. systemic or environmental) causes of pulmonary fibrosis in young patients (particularly females) [2]. IPF is defined by a radiological and/or pathological pattern of UIP. However, a number of clinical conditions are also associated with a UIP pattern, including CTD (primarily rheumatoid arthritis), drug toxicity, chronic HP, asbestosis and Hermansky-Pudlak syndrome [1]. Thus, UIP is not synonymous with IPF, and diagnosis of IPF requires the thorough search for (and exclusion of) all known causes of pulmonary fibrosis (e.g. idiopathic UIP). Owing to the difficulties in diagnosing IPF in clinical practice, the diagnostic gold standard consists of a multidisciplinary approach involving chest physicians, radiologists and pathologists with expertise in interstitial lung disease (ILD). The added value of such an integrated approach is particularly

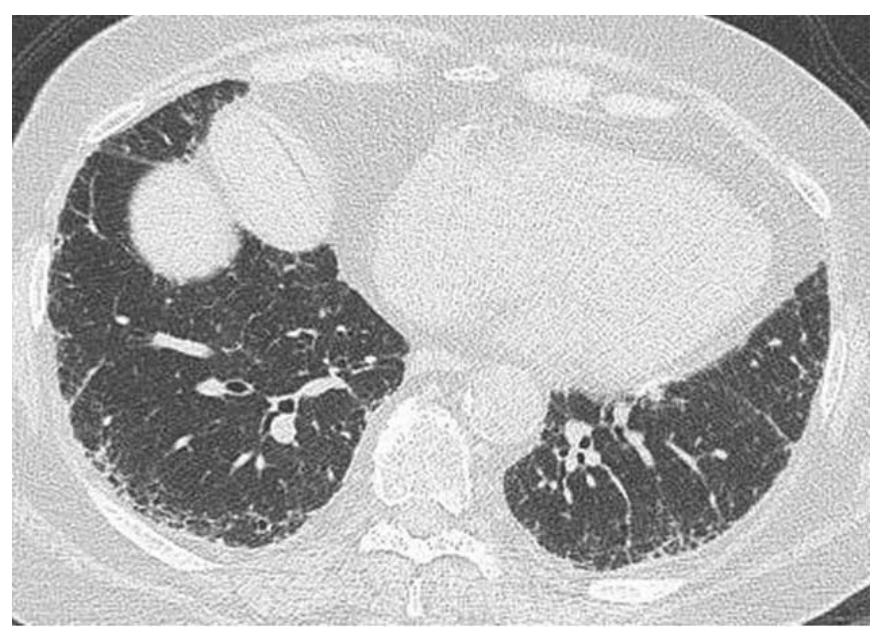

FIGURE 1 A possible usual interstitial pneumonia pattern. Computed tomography scan showing subpleural and bibasal predominant reticular abnormality without honeycombing. Although this patient was highly likely to have idiopathic pulmonary fibrosis (in the appropriate clinical setting), surgical lung biopsy is needed to confirm the diagnosis. 


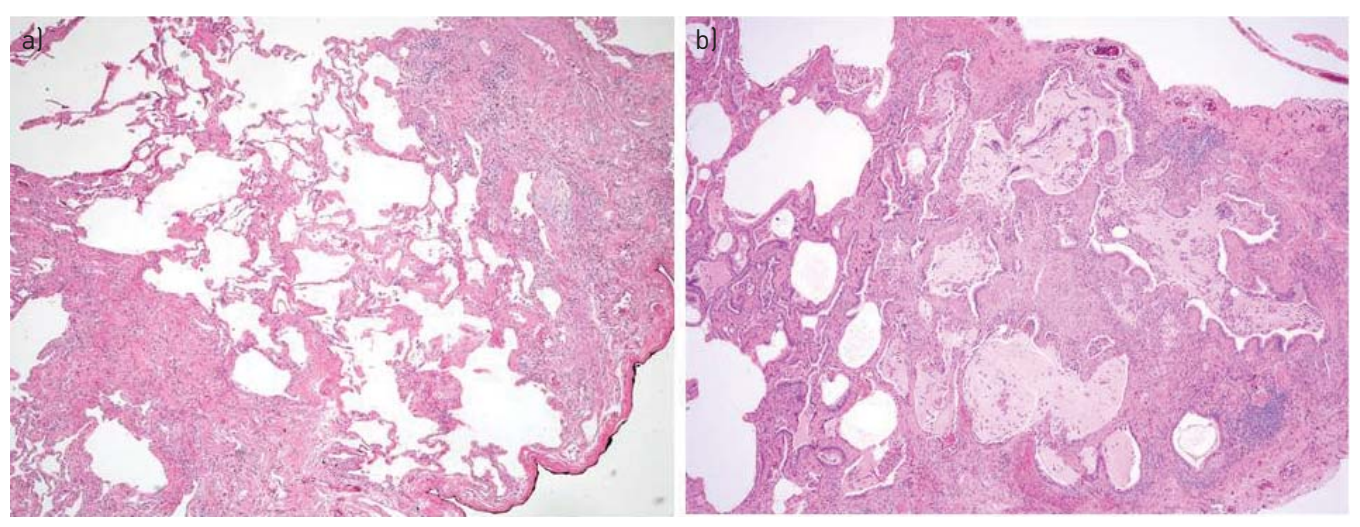

FIGURE 2 a) Open lung biopsy showing fibrotic lung disease with heterogeneous spatial and temporal fibrosis consistent with a usual interstitial pneumonia pattern (haematoxylin and eosin stain, $40 \times$ ). b) Honeycomb change with enlarged subpleural alveolar spaces with bronchiolar metaplasia, mucus plugs, and mild chronic inflammatory infiltrate (haematoxylin and eosin stain, $40 \times$ ).

important in cases with discordant radiological and histopathological abnormalities (e.g. HRCT inconsistent with UIP, but SLB suggestive of UIP).

\section{Delay in diagnosis}

Suspicion of IPF usually arises only several months after the onset of symptoms. Indeed, the initial manifestations of the disease (exertional dyspnoea and dry cough) tend to be initially overlooked and attributed to smoking habits or ageing [3]. Other common causes of diagnostic delay include reluctance of patients to undergo invasive procedures, thus, precluding obtaining tissue samples or, in the case of community-based physicians, insufficient expertise in ILD [4]. For instance, community-based physicians are more likely to formulate a final diagnosis of IPF (sometimes incorrectly) compared with academic physicians, highlighting the importance of referring patients with suspected IPF to expert centres [5]. In turn, timely referral of patients to an ILD specialist centre may allow institution of appropriate therapies, and access to clinical trials and lung transplant evaluation. Notably, a longer time from the onset of dyspnoea to the time of first evaluation at a subspecialty clinic is associated with an increased risk of death, independent of disease severity [6].

\section{Excluding known causes of pulmonary fibrosis}

In patients with suspected IPF, the most prevalent diagnostic difficulty is the exclusion of underlying conditions in which inflammation precedes and leads to fibrosis, primarily CTD and chronic HP. In our case, there was a family history of rheumatoid arthritis, and exposure to amiodarone and parrots. In a recent case-cohort study, almost half of the patients initially diagnosed with IPF based on the 2011 criteria were subsequently diagnosed with chronic HP following elicitation of a detailed history and application of a comprehensive diagnostic protocol [7]. Differentiating between the two has substantial therapeutic and prognostic implications. Indeed, immunosuppressive therapy, which is often appropriate in chronic HP (together with removal of the offending antigen, if identified), has been associated with increased risk of death in patients with IPF [8]. IPF and chronic HP can be indistinguishable. In our case, despite a history of exposure to avian antigens (which had ceased almost 20 years before symptom onset) and, thus, a high level of suspicion, there were no clear laboratory (serum precipitins or BAL lymphocytosis), radiological (ground-glass opacities, poorly defined centrilobular nodules, mosaic attenuation and air trapping, and lack of lower zone predominance on HRCT) or pathological (bronchiolocentric accentuation of the inflammation, peribronchial fibrosis, bronchiolar epithelial hyperplasia, and presence of granulomas or multinucleated giant cells) findings suggestive of a diagnosis of chronic HP [9]. ILD can be the sole clinical manifestation of CTD [10]. However, in our case there were no clinical, serological or pathological findings suggestive of an underlying systemic disease. Patients with IPF may have mildly positive anti-nuclear antibody and/or rheumatoid factor serology, although their frequency does not appear to differ from healthy, age-matched controls [11]. Finally, despite his exposure to amiodarone, we confidently excluded that our patient had a drug-induced ILD based on distinctive radiological, BAL and pathological findings.

\section{Determining when a confirmatory SLB is needed}

In clinical practice, SLB is performed in a minority of patients with suspected IPF. This is either due to the risks associated with the procedure, which may outweigh the benefits of establishing a secure diagnosis, 
or because of the reluctance of most patients to undergo surgery. In addition, impaired lung function, oxygenation and functional status, which are often present in these patients, increase the risk of postoperative complications, although improved surgical techniques have made the procedure much safer [12]. In the appropriate clinical setting (typically, male current or ex-smokers $>60$ years of age with unexplained exertional dyspnoea and pulmonary fibrosis of unknown cause), histological confirmation is not needed when a confident diagnosis is made by HRCT (e.g. the presence of a UIP pattern on HRCT has a high positive predictive value for the presence of a histological pattern of UIP). UIP is defined on computed tomography $(\mathrm{CT})$ as a peripheral and basal distribution of honeycomb changes with traction bronchiectasis, irregular interlobular septal thickening and minimal ground-glass opacity. When all these features are present, the diagnostic accuracy of CT approaches 90-100\%, with honeycombing being the strongest predictor of a diagnosis of UIP [13]. However, these abnormalities are only seen in around half of all patients with IPF [14].

A number of studies have evaluated the level of agreement between the clinical/radiological diagnosis and the final diagnosis reached after including histopathological data [14-17]. Overall, these studies demonstrate that HRCT has a high specificity for diagnosing UIP (confirmed by pathology), particularly in the setting of a high-confidence interpretation. By contrast, HRCT cannot rule out UIP, regardless of confidence [18]. As such, if the interpretation of CT data is anything other than definite UIP, as in our patient, SLB is warranted to establish the final diagnosis [19]. However, even patients with a modest amount of fibrosis on HRCT (reticular abnormalities and traction bronchiectasis without honeycombing) can be diagnosed as having IPF with almost 100\% certainty (i.e. without the need for a confirmatory SLB) if they fall into the older age group [20,21]. More recently, it has been suggested that in the appropriate clinical setting, a pattern of possible UIP on CT could be sufficient for a diagnosis of IPF to be established without SLB $[22,23]$. However, this study, which was a secondary analysis of a randomised controlled trial, excluded patients with radiological possible UIP and a SLB suggestive of an alternative diagnosis, mainly chronic HP and nonspecific interstitial pneumonia (NSIP), two of the most common (and difficult to differentiate) mimics of IPF.

Some clinical, laboratory and radiographic findings may obviate the need for SLB. For instance, patients with a history of extensive asbestos exposure, pleural plaques and typical CT abnormalities may be diagnosed with asbestosis without biopsy [24]. Clear environmental, occupational or drug exposures should raise the suspicion of HP, pneumoconioses or drug-related pulmonary toxicity, respectively. Similarly, in patients with pulmonary fibrosis and clinico-serological features suggestive of an underlying CTD, the diagnosis does not require lung biopsy. Usually, demographics and clinical history are inadequate in providing specific diagnostic clues. However, IPF is uncommon in individuals $<50$ years of age, except in rare cases of familial forms [25].

\section{Differential diagnosis of UIP pattern \\ Distinctive clinical features}

A complete clinical assessment is a key element in the diagnostic work-up of a patient with ILD. This includes a thorough history assessment, with careful evaluation of the main complaint; a comprehensive review of multiple systems; identification of all medications or drugs; and an exhaustive review of past medical, social, family and occupational histories with emphasis on potential environmental exposures. Similarly, a careful physical examination is essential. The clues that surface during this evaluation very often help clinicians to narrow the broad differential diagnosis to a few possible disorders [26].

\section{Idiopathic pulmonary fibrosis}

At presentation, virtually all patients complain of slowly progressive exertional dyspnoea, often associated with chronic dry cough. The median duration of symptoms before diagnosis is 24 months [27]. The incidence of the disease increases with older age [28]. In most patients, chest auscultation reveals endinspiratory fine crackles (velcro-like) prevalent at the lung bases. Finger clubbing occurs in approximately $40-75 \%$ of patients and is more common than in other ILDs [29, 30].

\section{Rheumatoid arthritis}

Rheumatoid arthritis is the most common CTD affecting almost $1 \%$ of the population. Despite a female-tomale ratio of three to one in rheumatoid arthritis, ILD in rheumatoid arthritis affects males twice as frequently as females [31]. The prevalence of ILD in rheumatoid arthritis ranges from $5 \%$ to $58 \%$ depending upon method of ascertainment and selection criteria [32]. Cigarette smoking and high titres of rheumatoid factor are well-established risk factors for the development of ILD in patients with rheumatoid arthritis [33]. In the majority of cases, articular manifestations precede the development of ILD, whereas interstitial lung abnormalities may be the presenting feature in approximately $10-20 \%$ of cases $[34,35]$. Recent data 
suggest that the prevalence of ILD in patients with rheumatoid arthritis increases with advancing age, and that the presence of a UIP pattern on HRCT carries a prognosis similar to IPF [36]. Patients with rheumatoid arthritis-associated ILD most commonly complain of progressive exertional dyspnoea (which can be initially masked by joint involvement and disability) and dry cough, whereas physical examination may reveal, in advanced cases, digital clubbing and velcro-like crackles [37]. From a clinical standpoint, younger age, articular or cutaneous manifestations and serological abnormalities are important discriminators between rheumatoid arthritis-associated ILD and IPF.

Hypersensitivity pneumonitis

HP, also termed extrinsic allergic alveolitis, is a form of diffuse parenchymal lung disease resulting from an aberrant immunological response to (mostly organic) inhaled allergens in susceptible individuals [38]. The prevalence of HP ranges widely around the world due to a number of factors, including disease definition, diagnostic methods, type and intensity of exposure, geographical conditions, agricultural and industrial practices, and host risk factors [9]. In addition, the disease is often unrecognised or misdiagnosed, which further complicates determining its exact prevalence. Smoking is less prevalent in patients with HP compared with controls under the same risk of exposure and appears to protect against the development of the disease by impairing macrophage activation, lymphocyte proliferation and T-cell function [39]. Conventionally, three forms of HP are identified (acute, subacute or chronic, mostly depending on the frequency and intensity of antigen exposure), but they may overlap both clinically and radiologically, and it is uncertain whether they represent different stages of the disease [40]. In chronic HP, the onset of symptoms is often insidious with slowly progressive dyspnoea on exertion, dry cough, fatigue, malaise and weight loss. Clinical examination may reveal bibasilar crackles whereas digital clubbing is seen in $20-50 \%$ of cases [41]. A rather unique clinical finding in chronic HP, in contrast to other chronic fibrotic ILDs, is the presence of inspiratory "squeaks", which are caused by coexisting bronchiolitis. Lung function tests typically reveal a restrictive ventilatory defect and impaired gas exchange. The presence of a UIP pattern of fibrosis, either on lung biopsy or HRCT, carries a survival rate similar to that observed in IPF [42]. The diagnosis of HP requires proven or suspected exposure associated with exposure-related symptoms; proof of sensitisation (e.g. demonstration of serum precipitins or lymphocytosis in the BAL fluid); and compatible chest radiography/HRCT abnormalities (with or without restrictive ventilatory/gas exchange defects) [41]. However, serum precipitating antibodies to a variety of common causative antigens have low sensitivity and specificity, and are helpful when positive but not if negative. Chronic HP may represent a diagnostic dilemma, particularly when a detailed clinical history does not reveal a temporal relationship between symptom onset and antigen exposure.

\section{Amiodarone pulmonary toxicity}

Amiodarone is a iodine-containing agent commonly used for the treatment of supraventricular and ventricular arrhythmias. This drug has been associated with a variety of adverse events, the most serious being lung toxicity, which can result either from a direct (cytotoxic) or indirect (immune-mediated) mechanism [43]. The term amiodarone pulmonary toxicity (APT) refers to a variety of distinct clinical, radiological and pathological patterns of lung involvement exhibiting a wide range of severity and outcome [44]. Although lung toxicity can occur at any time after treatment is initiated, individuals who have received a daily dose $\geqslant 400 \mathrm{mg}$ for more than 2 months or a lower dose (e.g. $200 \mathrm{mg}$ daily) for more than 2 years are considered at greatest risk. The frequency of APT is higher in men and increases with age. Pre-existing lung disease, cardiothoracic surgery and exposure to high concentrations of oxygen appear to represent additional risk factors for the development of APT [45]. Most commonly, patients with APT present with progressive shortness of breath, unproductive cough, malaise, fever and occasionally pleuritic chest pain of subacute onset. Physical examination may be unremarkable in milder cases, whereas diffuse crackles, hypoxaemia and respiratory distress may be noted in severe cases [46]. Lung function tests usually reveal a restrictive ventilatory pattern with reduced DLCO. Rarely, APT may manifest dramatically as acute respiratory failure and imaging typical of acute respiratory distress syndrome [47, 48]. Pulmonary fibrosis develops in $5-10 \%$ of cases, and may or may not be preceded by typical amiodarone pneumonitis [45]. APT should be suspected in any patient taking amiodarone who has new or worsening respiratory symptoms and/or new infiltrates on chest radiography. Open lung biopsy is rarely necessary to confirm the diagnosis and should be limited to highly selected cases because of the tendency for APT to worsen after thoracic surgery, and because such patients usually have impaired cardiac and pulmonary function.

\section{Radiological features}

IPF can be diagnosed in a significant proportion of cases based on clinical and imaging data, without the need for a surgical biopsy, because of the high diagnostic precision of CT [49]. However, a confident radiological diagnosis of UIP is only possible in about half of the cases, mainly because of the uncertainty in 
identifying honeycombing, the distinguishing feature of radiological UIP [15, 50]. Indeed, abnormalities such as cysts or confluence of traction bronchiectasis may erroneously be interpreted as honeycombing [51]. As such, because several conditions may display a HRCT pattern similar to UIP, radiologists should always indicate their level of diagnostic confidence and use all available tools (e.g. multiplanar reconstruction and comparison with previous examinations, if available) in order to discriminate between honeycombing, traction bronchiectasis or bronchiolectasis, and subpleural paraseptal emphysema.

Although data from population-based studies are not available, CT features of a possible UIP pattern are frequently encountered in clinical practice. Such cases should be carefully reviewed as they may represent fibrotic NSIP or chronic HP (and not IPF). For instance, while reticulation may look peripheral in fibrotic NSIP, its relative subpleural sparing is a radiographic hint that should not be overlooked. Chronic HP may also present with patchy subpleural reticulation, but usually with a mid-upper zone predilection (fig. 3). Other ancillary features commonly observed in HP include lobulated areas of decreased attenuation (indicative of air trapping) and centrilobular nodules. While these abnormalities may also be observed in smokers with IPF (indicative of constrictive-like bronchiolitis and respiratory bronchiolitis, respectively), when sufficiently profuse they are good discriminators for a diagnosis of chronic HP. SiLVA et al. [52] assessed the accuracy of HRCT in differentiating chronic HP from IPF and NSIP. Lobular areas with decreased attenuation were seen in $80 \%$ of patients with chronic HP, but only in $43 \%$ and $34 \%$ of those with IPF and NSIP, respectively. Similarly, centrilobular nodules were more frequently observed in chronic HP (56\%) compared with IPF (15\%) and NSIP (14\%) patients. Thin-walled cysts were also more common in chronic HP $(39 \%)$ than in IPF $(0 \%)$ or NSIP $(12 \%)$. Notably, the frequency of honeycombing was similar in chronic HP (64\%) and IPF (67\%), although patients with IPF were more likely to have basal predominance of honeycombing and fibrosis (52\%) than those with chronic HP (11\%).

A UIP pattern may also be observed in patients with CTD, particularly in the context of rheumatoid arthritis. TOKURA et al. [53] have reported a higher frequency of air trapping on expiratory CT scanning in rheumatoid arthritis-related ILD than in IPF, which may reflect coexisting airway disease. In addition, SONG et al. [54] have shown that patients with rheumatoid arthritis are more likely to have a nontypical UIP pattern (e.g. without honeycombing) compared with IPF. Nevertheless, the UIP pattern observed in rheumatoid arthritis may be virtually identical to that of IPF, especially in the absence of airways abnormalities, such as fuzzy centrilobular nodules, ground-glass opacities, or bronchial and bronchiolar wall thickening with heterogeneous lung attenuation [55]. By contrast, emphysema may be observed in both rheumatoid arthritis associated-ILD and IPF. In a recent study, emphysema was present in 66 (35\%) out of 186 patients with IPF and $22(48 \%)$ out of 46 smokers with rheumatoid arthritis associated-ILD, suggesting a possible pathogenetic link to smoking in both diseases [56].

The spectrum of abnormalities seen on CT in patients with drug-induced lung disease mirrors the underlying histopathology, which includes diffuse alveolar damage, NSIP, organising pneumonia, eosinophilic pneumonia and pulmonary haemorrhage [57]. By contrast, a radiological pattern of UIP is

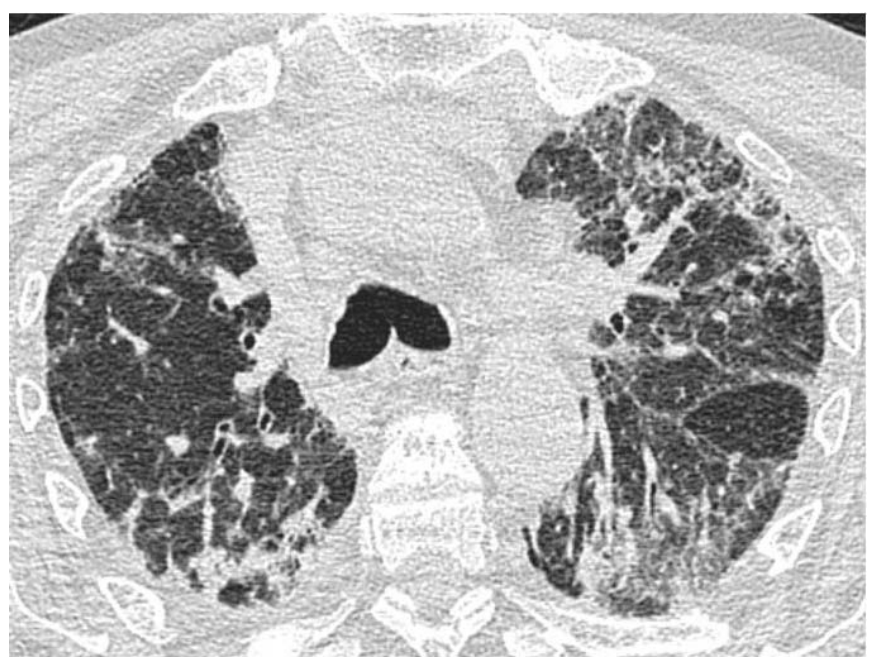

FIGURE 3 Chronic hypersensitivity pneumonitis with the computed tomography scan showing extensive reticular and ground-glass opacities. Traction bronchiectasis is also present in the apical segment of the left lower lobe. The left fissure is pulled apart because of the underlying fibrosis. 
infrequently seen in these conditions. Amiodarone-induced pulmonary fibrosis usually manifests on CT as interstitial and perilobular reticular opacities with basal predominance and areas of ground-glass opacity, consistent with a pattern of fibrotic NSIP, although architectural distortion and honeycombing may also be observed [58]. In the appropriate clinical setting, CT appearances may be virtually diagnostic of amiodarone-induced ILD by virtue of the high-attenuation pulmonary infiltrates thought to be caused by the iodinated properties of the drug and its prolonged half-time in the lung [44].

\section{UIP pattern on histology: considerations for the clinician}

Historically, a UIP pattern on biopsy has been the key element in the diagnosis of IPF. However, in the past few years the limitations of histology have been better appreciated, particularly interobserver variation among pathologists and sampling error [59]. For example, areas of fibrotic NSIP are frequently present in UIP, and if the wrong area is sampled an erroneous histological diagnosis of NSIP can be made. However, the prognosis will depend on the nonsampled UIP [60, 61]. For the pathologist examining a biopsy from a patient with fibrotic ILD, the main task is to differentiate UIP pattern from several mimickers. Once UIP is recognised, the second goal is to help the clinician to differentiate idiopathic UIP (e.g. IPF) from UIP secondary to an underlying cause or systemic disorder.

\section{The histology of UIP pattern}

The key histology features of the UIP pattern are: 1) spatial heterogeneity due to patchy parenchymal involvement; 2) architectural distortion; and 3) temporal heterogeneity (fig. 4) [62, 63]. At low magnification, spatial heterogeneity appears as patchy parenchymal involvement with abrupt transition from normal to diseased lung. The architecture is distorted, with scars and honeycomb changes obliterating the normal alveolar tissue. Honeycombing consists of enlarged airspaces embedded in fibrotic tissue, lined by bronchiolar epithelium and often filled with mucin and inflammatory cells. Honeycombing can be absent in early disease. Temporal heterogeneity is due to the presence of fibroblastic foci, which are the site of ongoing injury, embedded in a background of scar tissue and honeycombing, which are indicative of an old established lung injury. Fibroblastic foci consist of dome-shaped collections of spindle-shaped fibroblasts and myofibroblasts within myxoid stroma. Inflammation is usually mild and is mainly confined to honeycombing areas.

\section{Secondary UIP}

The histology of "secondary" UIP can be identical to "idiopathic" UIP/IPF, and in these cases the distinction is mainly based on clinical and laboratory findings. Nevertheless, there are findings that, in the context of UIP, may suggest the possibility of an underlying CTD or chronic HP (table 1 and fig. 5) [64-67]. These abnormalities can be subtle and should be carefully searched for by the pathologist. However, none of these features are specific as they can be found also in IPF, although less frequently. As such, the final diagnosis should rest on the correlation of histological findings with clinical and radiological data.

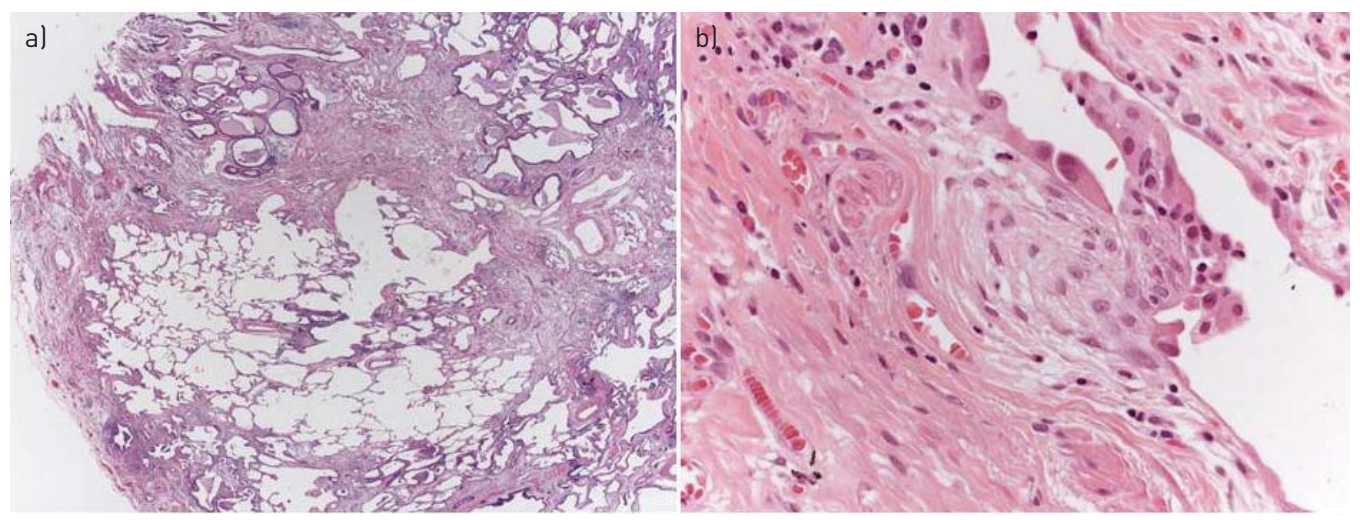

FIGURE 4 Usual interstitial pneumonia pattern in two patients with idiopathic pulmonary fibrosis. a) At low magnification, subpleural/paraseptal scars obscuring the alveolar architecture alternate with normal lung ("patchy fibrosis"). At the top, two areas of honeycombing can be observed (haematoxylin and eosin stain, 20×). b) At higher magnification a fibroblastic focus, consisting of a dome-shaped collection of fibroblasts/myofibroblasts embedded in a myxoid matrix and covered by hyperplastic pneumocytes, is observed. Note the pale grey colour of the fibroblastic focus, contrasting with the pink colour of the underlying scar (haematoxylin and eosin stain, $400 \times$ ). 
TABLE 1 Histological clues that, when present in the usual interstitial pneumonia pattern, suggest the possibility of an underlying connective tissue disease (CTD) or chronic hypersensitivity pneumonitis (HP)

Feature

Cellular (lymphocytic and/or plasmacellular) interstitial infiltrate

Plasma cells

Cellular bronchiolitis

Centrilobular fibrosis, with or without bridging fibrosis between bronchioles and pleura

Pleuritis

Small interstitial/peribronchiolar granulomas

Coexistence of more than one pattern in the same biopsy
CTD

Chronic HP

+: positive; -: negative. ${ }^{\#}$ : except in Sjögren's syndrome, in which small granulomas can be found.

Connective tissue disease

CTD may display a heterogeneous spectrum of histological abnormalities in the lungs. While in the majority of cases, the histopathology is that of NSIP, a UIP pattern may also be seen, particularly in rheumatoid arthritis and Sjögren's syndrome $[55,68]$ The presence of florid follicular bronchiolitis with germinal centres or prominent interstitial lymphocytic infiltrate on a background of fibrotic lung with a UIP pattern suggests a diagnosis of rheumatoid arthritis and Sjögren's syndrome, respectively [34, 65, 69]. Another helpful distinguishing feature is the presence of overlapping acute, subacute and chronic changes in the same biopsy when CTD produces pulmonary fibrosis. In addition, patients with CTD-UIP have fewer fibroblastic foci, smaller honeycomb cysts and less emphysema than patients with IPF [54]. Finally, pleural fibrosis is typically present in rheumatoid arthritis and other CTDs with thoracic manifestations but is uncommon in IPF [55].

Chronic HP

As expected in a disease that results from repeated exposure to inhaled antigens, most cases of chronic HP are characterised by airway-centred fibrosis. However, occasionally, chronic HP may manifest as established fibrosis indistinguishable from the UIP pattern observed in IPF. A number of histopathological features may help suggest chronic HP as the aetiology. They include: 1) an upper lobe-predominant UIP pattern; 2) the presence of "bridging" fibrosis, i.e. linear connection between centrilobular and perilobular areas (subpleural and/or paraseptal), or between centrilobular and adjacent centrilobular areas; 3 ) the presence of interstitial (not intra-alveolar) isolated giant cells or granulomas; and 4) cellular bronchiolitis with bronchiolocentric damage and peribronchiolar metaplasia (so-called lambertosis) [64]. These abnormalities can be subtle, thus requiring a meticulous examination on serial sections.

\section{Amiodarone pulmonary toxicity}

On histology, APT is characterised by an acute or subacute alveolar injury with hyperplastic type 2 pneumocytes and intra-alveolar foamy macrophages [45]. Bronchiolitis obliterans organising pneumonia and diffuse alveolar damage may also be observed. Type 2 reactive pneumocytes have a peculiar foamy cytoplasm.
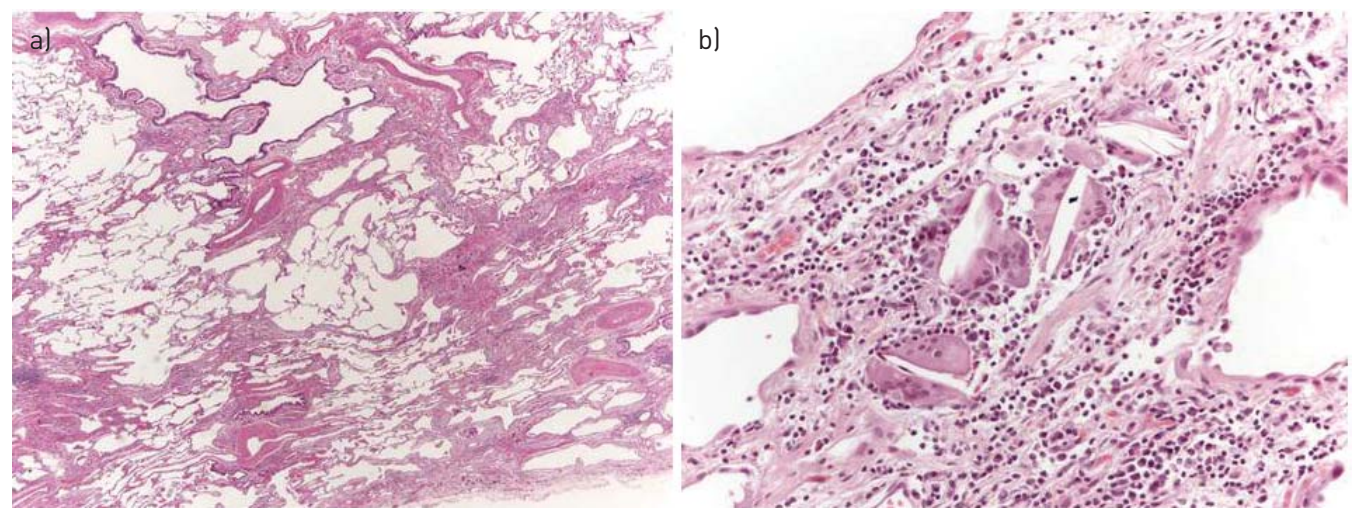

FIGURE 5 Chronic hypersensitivity pneumonitis with usual interstitial pneumonia pattern. a) At low magnification, bronchiolar damage with bridging fibrosis between bronchiole and pleura are the clues suggesting chronic hypersensitivity pneumonitis (haematoxylin and eosin stain, $20 \times$ ). b) At higher magnification, a few inconspicuous interstitial granulomas, consisting of isolated giant cells with cholesterol clefts, can be observed (haematoxylin and eosin stain, $200 \times$ ). 
Since amiodarone toxicity is characterised by an alveolar pattern of disease, the abnormalities observed in a SLB may potentially also be seen in a transbronchial biopsy, although unusual histological manifestations have also been reported [70, 71].

\section{Role of BAL in the differential diagnosis}

In most ILDs, BAL findings are not specific and do not allow a secure diagnosis as a stand-alone test. However, when BAL cellular analysis is considered in the context of clinical and HRCT findings, it may contribute to narrowing the differential diagnosis and to avoiding open lung biopsy [72]. Up to 8\% of patients with an HRCT pattern of UIP have BAL findings suggestive of an alternative diagnosis [73].

The typical BAL profile in IPF is characterised by a moderately increased neutrophil count (10-30\% of the total cells) with or without an increase in eosinophil count. Usually, the neutrophil count is twice as high as the eosinophil count [74]. In total, 70-90\% of the patients show an increased neutrophil count, while $40-60 \%$ also show an increased eosinophil count. In $10-20 \%$ of the patients, a moderately increased lymphocyte count (proportion $<30 \%$ ) may additionally be seen. An isolated marked increase in lymphocytes is inconsistent with IPF. If this is observed, other diseases that are associated with BAL lymphocytosis need to the excluded [73].

The reported BAL findings in rheumatoid arthritis are highly variable and nonspecific. An increase in either or both lymphocytes and granulocytes can be seen [75-78]. Overall, lymphocytes appear to be a more prominent feature in rheumatoid arthritis than in systemic sclerosis. HP shows the highest total cell count and the highest lymphocyte count of all ILDs [74]. The total cell yield is usually very high ( $>20$ million from a 100-mL BAL), with the proportion of lymphocytes exceeding 50\% [74]. In patients with chronic HP presenting with the radiological aspects of fibrotic UIP or NSIP, the increase in BAL lymphocytes may be less prominent [79]. By contrast, even asymptomatic sensitised individuals may show increased lymphocytes in BAL fluid [79]. The number of activated T-cells is also increased, whereas the CD4/CD8 ratio may be decreased, increased or normal $[80,81]$. The ratio is usually higher in chronic than in acute or subacute HP [80]. Alveolar macrophages often show a foamy cytoplasm. Plasma cells can be seen in patients with recent antigen exposure [74]. During an acute episode of HP, the neutrophil count may increase transiently [74]. Notably, a normal BAL cell count or an isolated increase in neutrophils or eosinophils virtually excludes HP [79].

A multitude of drugs can induce an interstitial lung reaction, which may be either toxic or immunemediated. In addition, any type of alveolitis may be present in BAL (lymphocytic, neutrophilic, eosinophilic or mixed), as well as diffuse alveolar haemorrhage [82]. The most frequent pattern is a lymphocytic alveolitis with a predominance of $\mathrm{CD}^{+} \mathrm{T}$-cells. In APT, the presence of alveolar macrophages with a finely vacuolated foamy cytoplasm is characteristic, corresponding to a form of phospholipidosis. Foamy macrophages, which indicate exposure to amiodarone and not necessarily toxicity, can also be seen in HP and organising pneumonia as well as in individuals without clinical signs of ILD [82]. Nevertheless, the absence of foamy macrophages makes the diagnosis of APT highly unlikely. However, a normal BAL does not exclude the diagnosis of APT [83-85].

\section{Concluding remarks}

When dealing with a patient with ILD, the most important task is to diagnose UIP and to separate idiopathic UIP (the clinical correlate of IPF) from secondary UIP. In fact, while IPF, by definition, has UIP as its radiological-pathological correlate, the UIP pattern can be seen in a number of conditions, mainly CTD and chronic HP, although expert evaluation often reveals important distinguishing features. Because these entities have different mechanisms of injury, approach and response to therapy, and prognosis, making the correct diagnosis is vital. Yet, in clinical practice, this is a difficult task as in many patients, like the one we have described, several confounding factors may coexist. It is in such cases that integration of clinical, radiological and pathological data is most needed and most productive.

\section{Acknowledgements}

We thank Ulrich Costabel (Interstitial and Rare Lung Disease Unit, Ruhrlandklinik, University Hospital, University of Duisburg-Essen, Germany) for critical review of the manuscript and Dominique Spirig (InterMune International AG, Muttenz, Switzerland) for editorial assistance.

\section{References}

Raghu G, Collard HR, Egan JJ, et al. An official ATS/ERS/JRS/ALAT statement: idiopathic pulmonary fibrosis: evidence-based guidelines for diagnosis and management. Am J Respir Crit Care Med 2011; 183: 788-824.

2 Noble PW, Barkauskas CE, Jiang D. Pulmonary fibrosis: patterns and perpetrators. J Clin Invest 2012; 122: 2756-2762.

3 Spagnolo P, Du Bois RM, Cottin V. Rare lung disease and orphan drug development. Lancet Respir Med 2013; 1: $479-487$. 
Spagnolo P, Tonelli R, Cocconcelli E, et al. Idiopathic pulmonary fibrosis: diagnostic pitfalls and therapeutic challenges. Multidiscip Respir Med 2012; 7: 42.

5 Flaherty KR, Andrei AC, King TE Jr, et al. Idiopathic interstitial pneumonia: do community and academic physicians agree on diagnosis? Am J Respir Crit Care Med 2007; 175: 1054-1060.

6 Lamas DJ, Kawut SM, Bagiella E, et al. Delayed access and survival in idiopathic pulmonary fibrosis: a cohort study. Am J Respir Crit Care Med 2011; 184: 842-847.

7 Morell F, Villar A, Montero MÁ, et al. Chronic hypersensitivity pneumonitis in patients diagnosed with idiopathic pulmonary fibrosis: a prospective case-cohort study. Lancet Respir Med 2013; 1: 685-694.

8 Idiopathic Pulmonary Fibrosis Clinical Research Network, Raghu G, Anstrom KJ, et al. Prednisone, azathioprine, and N-acetylcysteine for pulmonary fibrosis. N Engl J Med 2012; 366: 1968-1977.

9 Selman M, Pardo A, King TE Jr. Hypersensitivity pneumonitis: insights in diagnosis and pathobiology. Am J Respir Crit Care Med 2012; 186: 314-324.

10 Fischer A, du Bois R. Interstitial lung disease in connective tissue disorders. Lancet 2012; 380: 689-698.

11 Lee JS, Kim EJ, Lynch KL, et al. Prevalence and clinical significance of circulating autoantibodies in idiopathic pulmonary fibrosis. Respir Med 2013; 107: 249-255.

12 Lettieri CJ, Veerappan GR, Helman DL, et al. Outcomes and safety of surgical lung biopsy for interstitial lung disease. Chest 2005; 127: 1600-1605.

13 Hunninghake GW, Lynch DA, Galvin JR, et al. Radiologic findings are strongly associated with a pathologic diagnosis of usual interstitial pneumonia. Chest 2003; 124: 1215-1223.

14 Hunninghake GW, Zimmerman MB, Schwartz DA, et al. Utility of a lung biopsy for the diagnosis of idiopathic pulmonary fibrosis. Am J Respir Crit Care Med 2001; 164: 193-196.

15 Flaherty KR, Thwaite EL, Kazerooni EA, et al. Radiological versus histological diagnosis in UIP and NSIP: survival implications. Thorax 2003; 58: 143-148.

16 Raghu G, Mageto YN, Lockhart D, et al. The accuracy of the clinical diagnosis of new-onset idiopathic pulmonary fibrosis and other interstitial lung disease: a prospective study. Chest 1999; 116: 1168-1174.

17 Sumikawa H, Johkoh T, Colby TV, et al. Computed tomography findings in pathological usual interstitial pneumonia: relationship to survival. Am J Respir Crit Care Med 2008; 177: 433-439.

18 Sverzellati N, Wells AU, Tomassetti S, et al. Biopsy-proved idiopathic pulmonary fibrosis: spectrum of nondiagnostic thin-section CT diagnoses. Radiology 2010; 254: 957-964.

19 Schmidt SL, Sundaram B, Flaherty KR. Diagnosing fibrotic lung disease: when is high-resolution computed tomography sufficient to make a diagnosis of idiopathic pulmonary fibrosis? Respirology 2009; 14: 934-939.

20 du Bois RM. An earlier and more confident diagnosis of idiopathic pulmonary fibrosis. Eur Respir Rev 2012; 21: $141-146$

21 Fell CD, Martinez FJ, Liu LX, et al. Clinical predictors of a diagnosis of idiopathic pulmonary fibrosis. Am J Respir Crit Care Med 2010; 181: 832-837.

22 Johannson KA, de Boer K, Wolters PJ, et al. Diagnosis of idiopathic pulmonary fibrosis with high-resolution CT. Lancet Respir Med 2014; 2: e5.

23 Raghu G, Lynch D, Godwin JD, et al. Diagnosis of idiopathic pulmonary fibrosis with high-resolution CT in patients with little or no radiological evidence of honeycombing: secondary analysis of a randomised, controlled trial. Lancet Respir Med 2014; 2: 277-284.

24 American Thoracic Society. Diagnosis and initial management of nonmalignant diseases related to asbestos. Am $J$ Respir Crit Care Med 2004; 170: 691-715.

25 Steele MP, Speer MC, Loyd JE, et al. Clinical and pathologic features of familial interstitial pneumonia. Am J Respir Crit Care Med 2005; 172: 1146-1152.

26 Raghu G, Brown KK. Interstitial lung disease: clinical evaluation and keys to an accurate diagnosis. Clin Chest Med 2004; 25: 409-419.

27 King TE Jr, Tooze JA, Schwarz MI, et al. Predicting survival in idiopathic pulmonary fibrosis: scoring system and survival model. Am J Respir Crit Care Med 2001; 164: 1171-1181.

28 Gribbin J, Hubbard RB, Le Jeune I, et al. Incidence and mortality of idiopathic pulmonary fibrosis and sarcoidosis in the UK. Thorax 2006; 61: 980-985.

29 Kanematsu T, Kitaichi M, Nishimura K, et al. Clubbing of the fingers and smooth-muscle proliferation in fibrotic changes in the lung in patients with idiopathic pulmonary fibrosis. Chest 1994; 105: 339-342.

30 Turner-Warwick M, Burrows B, Johnson A. Cryptogenic fibrosing alveolitis: clinical features and their influence on survival. Thorax 1980; 35: 171-180.

31 Antin-Ozerkis D, Evans J, Rubinowitz A, et al. Pulmonary manifestations of rheumatoid arthritis. Clin Chest Med 2010; 31: 451-478.

32 O'Dwyer DN, Armstrong ME, Cooke G, et al. Rheumatoid arthritis (RA) associated interstitial lung disease (ILD). Eur J Intern Med 2013; 24: 597-603.

33 Saag KG, Kolluri S, Koehnke RK, et al. Rheumatoid arthritis lung disease. Determinants of radiographic and physiologic abnormalities. Arthritis Rheum 1996; 39: 1711-1719.

34 Lee HK, Kim DS, Yoo B, et al. Histopathologic pattern and clinical features of rheumatoid arthritis-associated interstitial lung disease. Chest 2005; 127: 2019-2027.

35 Gochuico BR, Avila NA, Chow CK, et al. Progressive preclinical interstitial lung disease in rheumatoid arthritis. Arch Intern Med 2008; 168: 159-166.

36 Kim EJ, Elicker BM, Maldonado F, et al. Usual interstitial pneumonia in rheumatoid arthritis-associated interstitial lung disease. Eur Respir J 2010; 35: 1322-1328.

37 O’Donnell DE, Ora J, Webb KA, et al. Mechanisms of activity-related dyspnea in pulmonary diseases. Respir Physiol Neurobiol 2009; 167: 116-132.

38 Lacasse Y, Selman M, Costabel U, et al. Clinical diagnosis of hypersensitivity pneumonitis. Am J Respir Crit Care Med 2003; 168: 952-958.

39 Blanchet MR, Israël-Assayag E, Cormier Y. Inhibitory effect of nicotine on experimental hypersensitivity pneumonitis in vivo and in vitro. Am J Respir Crit Care Med 2004; 169: 903-909.

40 Glazer CS, Rose CS, Lynch DA. Clinical and radiologic manifestations of hypersensitivity pneumonitis. $J$ Thorac Imaging 2002; 17: 261-272. 


\section{Radiol 1992; 46: 232-236.}

59 Wells AU. Histopathologic diagnosis in diffuse lung disease: an ailing gold standard. Am I Respir Crit Care Med 2004; 170: 828-829.

60 Monaghan H, Wells AU, Colby TV, et al. Prognostic implications of histologic patterns in multiple surgical lung biopsies from patients with idiopathic interstitial pneumonias. Chest 2004; 125: 522-526.

61 Flaherty KR, Travis WD, Colby TV, et al. Histopathologic variability in usual and nonspecific interstitial pneumonias. Am J Respir Crit Care Med 2001; 164: 1722-1727.

62 Cavazza A, Rossi G, Carbonelli C, et al. The role of histology in idiopathic pulmonary fibrosis: an update. Respir Med 2010; 104: Suppl. 1, S11-S22.

63 Katzenstein AL, Mukhopadhyay S, Myers JL. Diagnosis of usual interstitial pneumonia and distinction from other fibrosing interstitial lung diseases. Hum Pathol 2008; 39: 1275-1294.

64 Takemura T, Akashi T, Kamiya H, et al. Pathological differentiation of chronic hypersensitivity pneumonitis from idiopathic pulmonary fibrosis/usual interstitial pneumonia. Histopathology 2012; 61: 1026-1035.

65 Trahan S, Hanak V, Ryu JH, et al. Role of surgical lung biopsy in separating chronic hypersensitivity pneumonia from usual interstitial pneumonia/idiopathic pulmonary fibrosis: analysis of 31 biopsies from 15 patients. Chest 2008; 134: 126-132.

66 Schneider F, Gruden J, Tazelaar HD, et al. Pleuropulmonary pathology in patients with rheumatic disease. Arch Pathol Lab Med 2012; 136: 1242-1252.

67 Churg A, Muller NL, Flint J, et al. Chronic hypersensitivity pneumonitis. Am J Surg Pathol 2006; 30: $201-208$.

68 Parambil JG, Myers JL, Lindell RM, et al. Interstitial lung disease in primary Sjögren syndrome. Chest 2006; 130: 1489-1495.

69 Nakamura Y, Suda T, Kaida Y, et al. Rheumatoid lung disease: prognostic analysis of 54 biopsy-proven cases. Respir Med 2012; 106: 1164-1169.

70 Larsen BT, Vaszar LT, Colby TV, et al. Lymphoid hyperplasia and eosinophilic pneumonia as histologic manifestations of amiodarone-induced lung toxicity. Am J Surg Pathol 2012; 36: 509-516.

71 Ruangchira-Urai R, Colby TV, Klein J, et al. Nodular amiodarone lung disease. Am J Surg Pathol 2008; 32: $1654-1660$.

72 Costabel U, Guzman J, Bonella F, et al. Bronchoalveolar lavage in other interstitial lung diseases. Semin Respir Crit Care Med 2007; 28: 514-524.

73 Ohshimo S, Bonella F, Cui A, et al. Significance of bronchoalveolar lavage for the diagnosis of idiopathic pulmonary fibrosis. Am J Respir Crit Care Med 2009; 179: 1043-1047.

74 Bonella F, Ohshimo S, Bauer P, et al. Bronchoalveolar lavage. In: Strausz J, Bolliger CT, eds. Interventional Pulmonology. Eur Respir Monogr 2010; 48: 59-72.

75 Garcia JG, Parhami N, Killam D, et al. Bronchoalveolar lavage fluid evaluation in rheumatoid arthritis. Am Rev Respir Dis 1986; 133: 450-454.

76 Popp W, Ritschka L, Scherak O, et al. Bronchoalveolar lavage in rheumatoid arthritis and secondary Sjögren's syndrome. Lung 1990; 168: 221-231.

77 Ishioka S, Inyaku K, Shirotani Y, et al. [Bronchoalveolar lavage fluid findings in rheumatoid arthritis]. Nihon Kyobu Shikkan Gakkai Zasshi 1992; 30: 614-618.

78 Balbi B, Cosulich E, Risso A, et al. The interstitial lung disease associated with rheumatoid arthritis: evidence for imbalance of helper T-lymphocyte subpopulations at sites of disease activity. Bull Eur Physiopathol Respir 1987; 23: $241-247$. 

537-556.

80 Barrera L, Mendoza F, Zuñiga J, et al. Functional diversity of T-cell subpopulations in subacute and chronic hypersensitivity pneumonitis. Am J Respir Crit Care Med 2008; 177: 44-55.

81 Ye Q, Nakamura S, Sarria R, et al. Interleukin 12, interleukin 18, and tumour necrosis factor alpha release by alveolar macrophages: acute and chronic hypersensitivity pneumonitis. Ann Allergy Asthma Immunol 2009; 102: 149-154.

82 Bonella F, Uzaslan E, Guzman J, et al. Bronchoalveolar lavage in drug-induced lung disease. In: Camus P, Rosenow EC, eds. Drug-induced and Iatrogenic Respiratory Disease. 1st Edn. London, CRC Press, 2010; pp. 32-42.

83 Akoun GM, Cadranel JL, Blanchette G, et al. Bronchoalveolar lavage cell data in amiodarone-associated pneumonitis. Evaluation in 22 patients. Chest 1991; 99: 1177-1182.

84 Ohar JA, Jackson F, Dettenmeier PA, et al. Bronchoalveolar lavage cell count and differential are not reliable indicators of amiodarone-induced pneumonitis. Chest 1992; 102: 999-1004.

85 Coudert B, Bailly F, Lombard JN, et al. Amiodarone pneumonitis. Bronchoalveolar lavage findings in 15 patients and review of the literature. Chest 1992; 102: 1005-1012. 